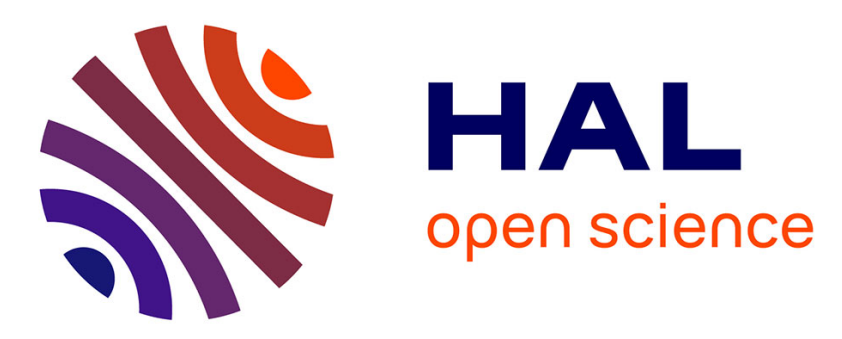

\title{
Multivariate Linear Time-Frequency modeling and adaptive robust target detection in highly textured monovariate SAR image
}

Jean-Philippe Ovarlez, Guillaume Ginolhac, Abdourrahmane Atto

\section{To cite this version:}

Jean-Philippe Ovarlez, Guillaume Ginolhac, Abdourrahmane Atto. Multivariate Linear TimeFrequency modeling and adaptive robust target detection in highly textured monovariate SAR image. 2017 IEEE International Conference on Acoustics, Speech and Signal Processing (ICASSP 2017), Mar 2017, New Orleans, United States. pp.4029 - 4033, 10.1109/ICASSP.2017.7952913 . hal-01617057

\section{HAL Id: hal-01617057 \\ https://hal.univ-grenoble-alpes.fr/hal-01617057}

Submitted on 16 Oct 2017

HAL is a multi-disciplinary open access archive for the deposit and dissemination of scientific research documents, whether they are published or not. The documents may come from teaching and research institutions in France or abroad, or from public or private research centers.
L'archive ouverte pluridisciplinaire $\mathbf{H A L}$, est destinée au dépôt et à la diffusion de documents scientifiques de niveau recherche, publiés ou non, émanant des établissements d'enseignement et de recherche français ou étrangers, des laboratoires publics ou privés. 


\title{
MULTIVARIATE LINEAR TIME-FREQUENCY MODELING AND ADAPTIVE ROBUST TARGET DETECTION IN HIGHLY TEXTURED MONOVARIATE SAR IMAGE
}

\author{
Jean-Philippe Ovarlez ${ }^{1,2}$, Guillaume Ginolhac ${ }^{3}$ and Abdourrahmane M. Atto ${ }^{3}$ \\ 1 CentraleSupélec/SONDRA, Plateau du Moulon, 3 rue Joliot-Curie, F-91190 Gif-sur-Yvette, France \\ 2 ONERA, DEMR/TSI, Chemin de la Hunière, F-91120 Palaiseau, France \\ 3 LISTIC, Université de Savoie Mont-Blanc, F-74944, Annecy le Vieux, France
}

\begin{abstract}
Usually, in radar imaging, the scatterers are supposed to respond the same way regardless of the angle from which they are viewed and have the same properties within the emitted spectral bandwidth. Nevertheless, new capacities in SAR imaging (large bandwidth, large angular extent) make this assumption obsolete. An original application of the Linear Time-Frequency Distributions (LTFD) in SAR imaging allows to highlight the spectral and angular diversities of these reflectors. This methodology allows to transform a monovariate SAR image onto multivariate SAR image. Robust detection schemes in Gaussian or non Gaussian background (Adaptive Matched Filter (AMF), Adaptive Normalized Matched Filter (ANMF), Anomaly Kelly Detector) associated with classical or robust Covariance Matrix Estimates (Sample Covariance Matrix (SCM), M-estimators) can then be applied exploiting these diversities. The combined two-methodologies show their very good performance for target detection.
\end{abstract}

Index Terms - Time-Frequency Distributions, Adaptive Normalized Match Filter, $M$-estimators, Tyler's estimator, Complex Elliptically Symmetric distributions, Non-Gaussian detection, Robust estimation theory.

\section{INTRODUCTION}

The Synthetic Aperture Radar (SAR) imaging process [1, 2] consists in acquiring and analyzing the backscattering coefficient $H(\mathbf{k})$ collected by a moving radar (see figure 1) and forming the 2D-spatial repartition $I(\mathbf{r})$ of the scatterers located in $\mathbf{r}=(x, y)^{T}$ which reflect a part of the emitted radar signal. The square modulus of $H(\mathbf{k})$ is called the Radar Cross Section of the scatterer for the wave vector $\mathbf{k}$. The wave vector $\mathbf{k}$ is related to the emitted frequency $f$ and to the direction $\theta$ of radar illumination by the relations $|\mathbf{k}|=2 \mathrm{f} / \mathrm{c}$, $\theta=\arg (\mathbf{k})$ where $c$ is the speed of light.

Conventional radar imaging models consider a target as a bright point. Indeed, they consider scatterers as isotropic for all the directions of presentation and decorrelated in the frequency band [3]. Whatever the pre-processing algorithm used (e.g.: the Range Migration Algorithm of [3]), the basic principle aims at deriving, from the acquired data, a focused bi-dimensional backscattering coefficient $H(\mathbf{k})$ for each illuminated point $\mathbf{r}$ on the ground and then, performing Fourier based spectral estimation in order to build the conventional complex single look (monovariate) SAR image $I(\mathbf{r})$ :

$$
I(\mathbf{r})=\int H(\mathbf{k}) \exp \left(2 i \pi \mathbf{k}^{T} \mathbf{r}\right) d \mathbf{k}
$$

where the integration is performed on the whole spectral and angular domains.

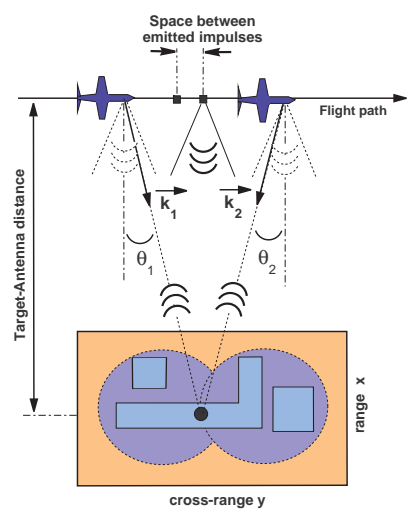

Fig. 1. A reflector, viewed at two different azimuthal angles of illumination in SAR-stripmap mode.

Target detection schemes in monovariate SAR image usually consist in analyzing statistics of the background surrounding the target to be detected and in performing an appropriate adaptive scalar thresholding operation (see for example the recent paper [4]). Amplitude pixel values which lie above the threshold are considered high and therefore likely to correspond to a dominant scatterer. This operation uses only the amplitude of the complex image: it does not exploit the additional diversities that are intrinsic to SAR imaging principle.

In this paper, we propose a detection framework that exploits spectral and angular diversities from a monovariate SAR image. In [5], these diversities have been considered for developing a matched subspace detector. However, the emitted electromagnetic wave is required in the processing, which results in a high computational time and can suffer from mismodeling. Diversities can also be created by using geometric image transforms such as steerable pyramids [6, 7] or curvelets [8], but the geometry under consideration in this transform is the natural one, not the one involved by SAR acquisition geometry. The contribution proposed by the paper is taking spectral and angular diversities into account from LTFD and SAR geometry. This methodology, presented in Section 2 allows to transform a single look complex SAR image onto multi-looks complex SAR images. Each pixel of the SAR image is then characterized by a vector of information related to its behavior in angular and frequency domains. Section 3 proposes to exploit these diversities by building robust adaptive detectors (AMF, ANMF) or Kelly-based anomaly detectors (Mahalanobis distance) associated with classical or robust covariance matrix estimates. These detectors are considered either 
for Gaussian or non-Gaussian background (textured SAR image). Section 4 provides experimental results performed on SAR images and Section 5 concludes the work.

\section{EXTENDED SAR IMAGE}

When an object is illuminated using a broad-band signal and/or for and a large angular extent, it is realistic to consider that the amplitude of the reflectors involves a dependence on frequency $f$ and aspect angle $\theta$. When considering Time-Frequency analysis, subspace image representations are called hyperimages and their characterisations of the angular, spectral and/or polarimetric behavior of the spatial distribution of scatterers in hyperimages can be found in [9, 10, 11, 12]. These characterisations show that some scatterer hyperimage features were neither isotropic nor decorrelated.

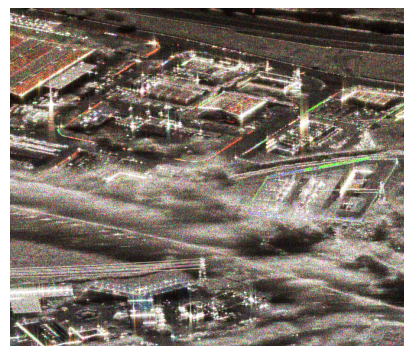

Fig. 2. An ONERA RGB color-coded SAR image acquired for three consecutive spectral sub-bands.

Figure 2 shows a ONERA SAR image of industrial area in Xband. The responses relative to three consecutive sub-bands have been highlighted in RGB color-coding. Red points are responding only on the first sub-band, green ones on the second sub-band and the blue ones on the third sub-band. They are called colored scatterers. Gray points are called white scatterers as they are responding equivalently in the three sub-bands. Angular diversity will show the same behavior (angular sub-looks SAR images). This spectral and angular non-stationary behavior of scatterers can be due to their material (dispersive), geometry (anisotropic and dispersive) or orientation (anisotropic). Such amplitude variation of scatterers has to be highlighted in order to see if this variation is potentially interpretable in terms of target characteristics. In this respect, this spectral and angular diversity should be exploited in any detector.

LTFD analysis and the physical group theory (Heisenberg or affine group) allow to construct hyperimages [13, 10, 11] through:

$$
\tilde{I}\left(\mathbf{r}_{0}, \mathbf{k}_{0}\right)=\int_{\mathcal{D}_{\mathbf{k}}} H(\mathbf{k}) \Psi_{\mathbf{r}_{0}, \mathbf{k}_{0}}^{*}(\mathbf{k}) d \mathbf{k}
$$

where $\Psi_{\mathbf{r}_{0}, \mathbf{k}_{0}}(\mathbf{k})$ is a family of wavelet bases (Gabor, standard wavelet) generated from a mother wavelet $\phi(\mathbf{k}, \arg \mathbf{k})$ through the chosen physical group of transformation (translations in frequency, rotation, similarity group, etc.) and where $\mathcal{D}_{\mathbf{k}}$ is the spectral/angular support of the wavelet $\Psi$.

The hyperimage can be interpreted as follows: for each emitted wave vector $\left|\mathbf{k}_{0}\right|$ (or equivalently for each frequency $f_{0}$ ) and each angle of illumination $\theta_{0}=\arg \mathbf{k}_{0}$, a spatial repartition $\tilde{I}\left(\mathbf{r}_{0}, \mathbf{k}_{0}\right)$ of reflectors which respond at this frequency and this angle can be defined. Conversely, for each reflector location $\mathbf{r}_{0}$, it is possible to analyse its behavior in spectral and angular spaces. Thereby, this extended SAR imaging with LTFD (linear meaning that the inphase and quadrature signal components have to be kept, in contrast
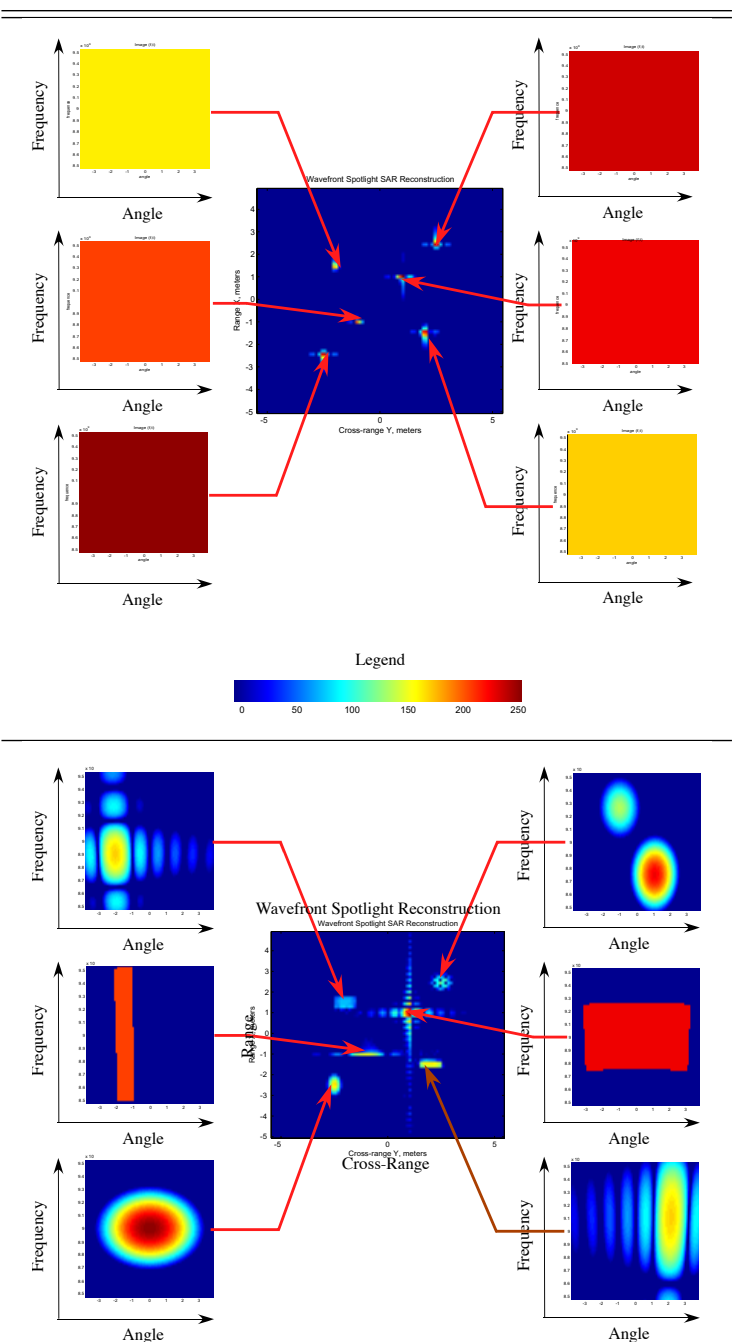

Legend

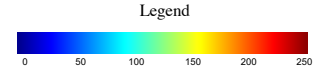

Fig. 3. Top: Example of theoretical model of isotropic and white scatterers. Bottom: Example of theoretical model of anisotropic and colored scatterers.

with energetic Bilinear TFD [14]) allows to construct hyperimages in four dimensions $(x, y, f, \theta)$ which depend on two free parameters $\left(\sigma_{f}, \sigma_{\theta}\right)$ which:

- control the spread of the mother wavelet $\phi(\mathbf{k}, \arg \mathbf{k})$ in spectral and angular domains and

- play an important role on interrelated resolutions in range, cross-range, frequency and angle.

Figure 3 shows the difference between the model of bright points (left) and those of colored and isotropic scatterers (right). This methodology allows to transform univariate SAR image onto multivariate SAR image and then brings angular and spectral diversities to each pixel of the SAR image [15]. Any SAR pixel can be characterized by a complex $N$-vector c containing angular and spectral information. In the following, we denote respectively by $N_{\theta}$ and $N_{f}$, the number of sub-looks and sub-bands characterizing the suc- 


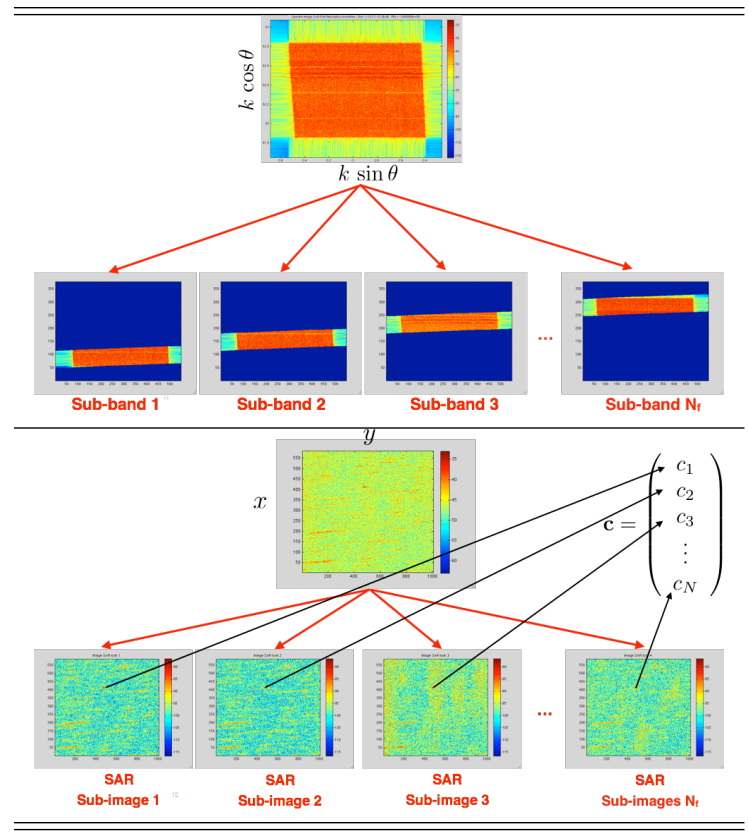

Fig. 4. Top: Example of $N_{f}$ frequency sub-bands decomposition of the backscattering coefficient $\left(N_{\theta}=1\right)$. Bottom: Corresponding sub-band SAR images: each pixel $(x, y)$ of the SAR image characterizes a complex vector $\mathbf{c}$ of information related to dispersion and anisotropy with dimension $N=N_{f} N_{\theta}$.

cessive positions of the mother wavelet, we have thus $N=N_{\theta} N_{f}$ (see Figure 4). This approach is very relevant because this diversity can be exploited to discriminate useful target from noise or background. This discrimination can be performed by modeling the background using multivariate statistics (Gaussian, Complex Elliptically Symmetric (CES) distributions) and by evaluating standard binary hypotheses tests. If the information (e.g. steering vector $\mathbf{p}$ ) related to the target is available, we can build adaptive detectors like AMF, ANMF. If no a priori information is available, Anomaly detectors based on the Mahalanobis distance and generally used for target detection in hyperspectral images [16, 17] can be exploited.

\section{DETECTION ON EXTENDED SAR IMAGE}

\subsection{Data Model}

In the following, each pixel of the SAR image will be represented by a set of LTFD features encapsulated in a random complex vector $\mathbf{c} \in \mathbb{C}^{N}$ where $N=N_{f} \times N_{\theta}$. For high resolution SAR images, it is well-known that the data cannot be correctly described by using only standard Gaussian statistics. Generalizing the Gaussian model assumption, we assume here that $\mathbf{c}$ follows a CES distribution $\mathcal{C} \mathcal{E}(\mathbf{0}, g, \mathbf{R})$ where the scatter matrix $\mathbf{R}$ is unknown and where $g$ stands for any characteristic function generator. This matrix $\mathbf{R}$ characterizes the angular and spectral behavior of each scatterer. To estimate this matrix, $K$ secondary data surrounding the pixel under test $\left\{\mathbf{c}_{k}\right\}_{k \in[1, K]}$ (supposed homogeneous in terms of angular and spectral behavior and generally obtained using a boxcar window centered around the target) can be used. Finally, as the Sample Covariance Matrix (SCM) is not adapted for a good estimation for any CES data, the Tyler's Estimator (TE) represents an alternative thanks to its good robustness property [18, 19]:

$$
\hat{\mathbf{R}}_{T E}=\frac{N}{K} \sum_{k=1}^{K} \frac{\mathbf{c}_{k} \mathbf{c}_{k}^{H}}{\mathbf{c}_{k}^{H} \hat{\mathbf{R}}_{T E}^{-1} \mathbf{c}_{k}} .
$$

The number $K$ has to be important with respect to $N$ for a good estimation. Otherwise, regularized versions of the TE exist in the literature [20, 21, 22].

\subsection{Detection schemes}

In this section, we assume that a target with a known steering vector $\mathbf{p} \in \mathbb{C}^{N}$ could be present in some pixels in the SAR image. In this case, we have for each pixel to solve the standard binary hypothesis test:

$$
\left\{\begin{array}{lll}
H_{0}: & \mathbf{c}=\mathbf{n}, & \mathbf{c}_{k}=\mathbf{n}_{k} \forall k \in[1, K] \\
H_{1}: & \mathbf{c}=a \mathbf{p}+\mathbf{n}, & \mathbf{c}_{k}=\mathbf{n}_{k} \forall k \in[1, K]
\end{array},\right.
$$

where $\mathbf{n}$ is the noise component in the cell under test $\mathbf{c}$, with $\left\{\mathbf{c}_{k}\right\}_{k \in[1, K]}$ being the $K$ secondary data and $a$ represents the unknown amplitude of the possible target.

\subsubsection{Adaptive Detectors}

From this detection problem, we decide to test different adaptive detectors like the well-known AMF (two-step GLRT in homogeneous Gaussian noise [23]):

$$
\hat{\Lambda}_{A M F}=\frac{\left|\mathbf{p}^{H} \hat{\mathbf{R}}^{-1} \mathbf{c}\right|^{2}}{\mathbf{p}^{H} \hat{\mathbf{R}}^{-1} \mathbf{p}} \underset{H_{0}}{\stackrel{H_{1}}{\gtrless}} \lambda,
$$

where $\hat{\mathbf{R}}$ generally stands for the SCM (Maximum Likelihood Estimate in Gaussian noise) and $\lambda$ is the detection threshold. For partially homogeneous Gaussian noise or for other CES noises, the AMF is known to provide a poor Constant False Alarm (FA) Rate and the ANMF is therefore more suitable [24 25]:

$$
\hat{\Lambda}_{A N M F}=\frac{\left|\mathbf{p}^{H} \hat{\mathbf{R}}^{-1} \mathbf{c}\right|^{2}}{\left(\mathbf{p}^{H} \hat{\mathbf{R}}^{-1} \mathbf{p}\right)\left(\mathbf{c}^{H} \hat{\mathbf{R}}^{-1} \mathbf{c}\right)} \underset{H_{0}}{\stackrel{H_{1}}{\gtrless}} \lambda .
$$

\subsubsection{Anomaly Detection}

When no information are available on the steering vector $\mathbf{p}$, considered as an unknown deterministic parameter, the Kelly-based GLRT approach proposed in [17] leads to the well-known Mahalanobis distance: $d_{m}=\mathbf{c}^{H} \hat{\mathbf{R}}^{-1} \mathbf{c}$, where $\hat{\mathbf{R}}^{-1}$ is estimated from $K$-secondary data (SCM or TE). When $\hat{\mathbf{R}}^{-1}$ is estimated on the basis of the $K$ secondary data, together with the cell under test, the GLRT problem leads to the well-known Reed-Xiaoli-Detector (so called RXD) [16]. This non-standard distance $d_{m}$ can be compared with a classical SPAN power measure $\mathbf{c}^{H} \mathbf{c}$.

\section{RESULTS}

Figure 5 shows a $400 \times 400$ SAR image available from Sandia National Laboratories (http://www.sandia.gov/radar/ complex_data /). In the analysis, we used $N_{\theta}=5$ sub-looks, $N_{f}=5$ sub-bands $(N=25)$ and $K=88$ secondary data coming from a $13 \times 13$ spatial mask with a $4 \times 4$ cell guards submask. We embedded in this image a colored and non-isotropic target with 

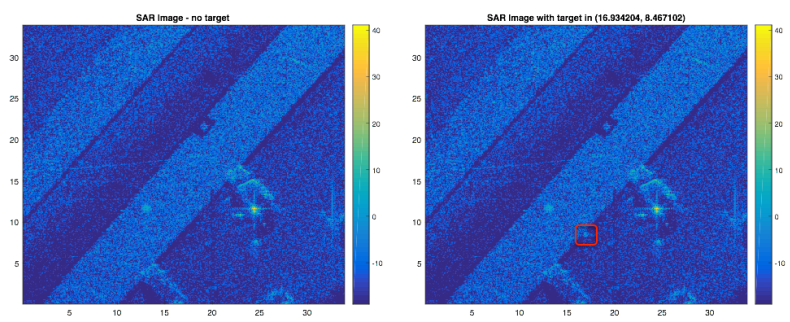

Fig. 5. Left: Original SAR Image without target. Right: SAR image with specific embedded target.
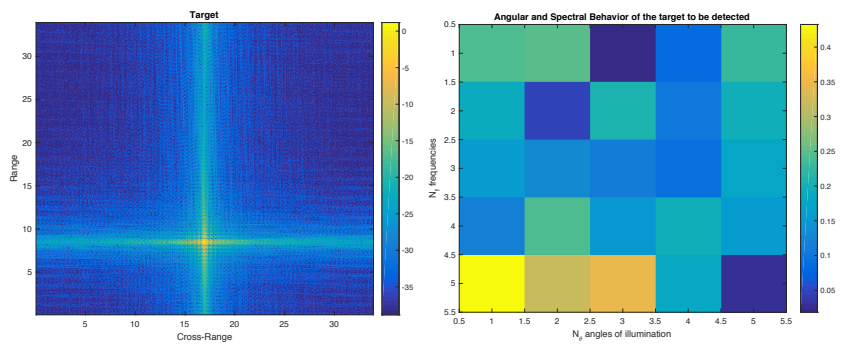

Fig. 6. Left: SAR Image of the target. Right: Reshaped target steering vector $\mathbf{p}$ in angular and spectral spaces.

spectral and angular behavior given in Figure 6 (the steering vector $\mathbf{p}$ of the target is chosen here as a fixed complex random vector). As it can be observed, these angular and spectral diversities defocuse the ideal point-like target in the pure SAR image. The SNR of the target is fixed to $-50 \mathrm{~dB}$. First, FA regulation has been analyzed.
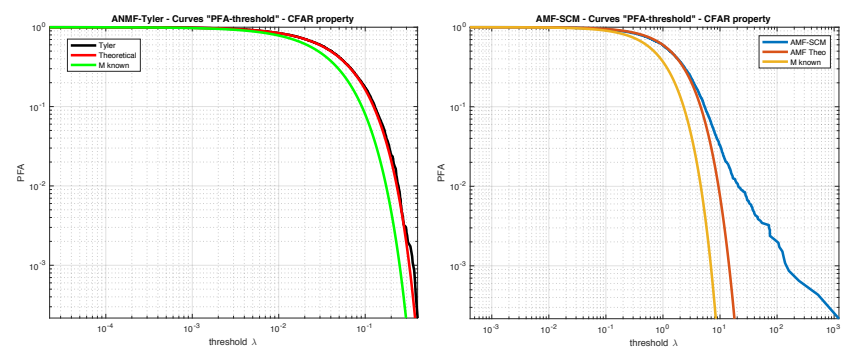

Fig. 7. Left: False AlarmFA Regulation with ANMF-Tyler. Right: FA Regulation with AMF-SCM. $N_{\theta}=5, N_{f}=5, K=88$.

The detection mask has been moved at each tested pixel under hypothesis $H_{0}$ (target not present). Figure 7 shows regulation of FA for the previous adaptive detectors (AMF-SCM and ANMF-TE). As expected, AMF-SCM has a poor behavior and does not reach the theoretical PFA-threshold relationship. The ANMF-TE shows a quite perfectly FA regulation. Figures 8 and 9 show the detection results for $P_{f a}=1$ (full dynamic of the test) and $P_{f a}=2.610^{-3}$. The AMF-SCM mainly focused on the power. Whatever the steering vector of the target to be detected, AMF-SCM detects another high targets while AMF-TE regulates better the FA and stays mainly focused on the target characterized by the steering vector $\mathbf{p}$ presented in figure 6. In Figure 10 the SPAN test does not allow to detect this quite weak target. Concerning the Figure 11 even if the PFA is set to low for detecting this target, the Mahalanobis distance test performs better than the SPAN and better regulates the clutter returns.
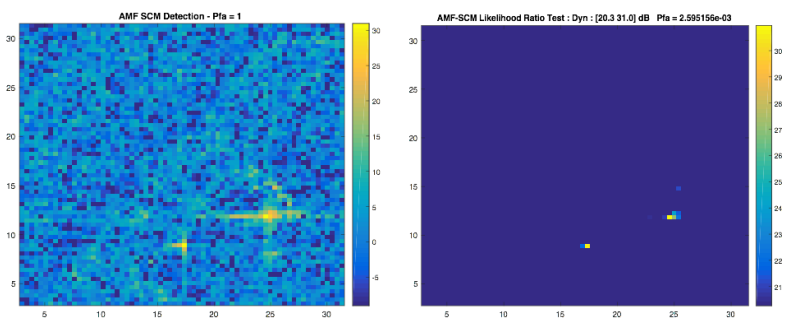

Fig. 8. Left: Full AMF -SCM detection test, $P_{f a}=1$. Right: Thresholded AMF -SCM detection dest, $P_{f a}=2.610^{-3}$.

\section{CONCLUSION AND FUTURES}

This paper focused on the Adaptive detection schemes with multivariate statistics (CES, M-estimates) in complex mono-look SAR image using LTFD. These tools allow to characterize angular and spectral diversities regarding the target and the background behaviors. Combined with recent Adaptive Detection Schemes (ANMF built with Tyler Estimate), the proposed approach allows to reach better performance than the classical thresholding detection schemes and classical AMF based on SCM. This diversity appears an important feature to improve target detection in textured images. This work will be extended to apply for polarimetric and interferometric SAR in future works.
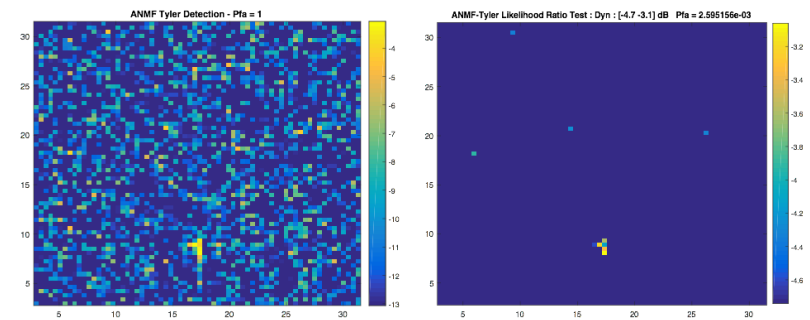

Fig. 9. Left: ANMF -TE detection test, $P_{f a}=1$. Right: Thresholded ANMF -TE detection test, $P_{f a}=2.610^{-3}$.
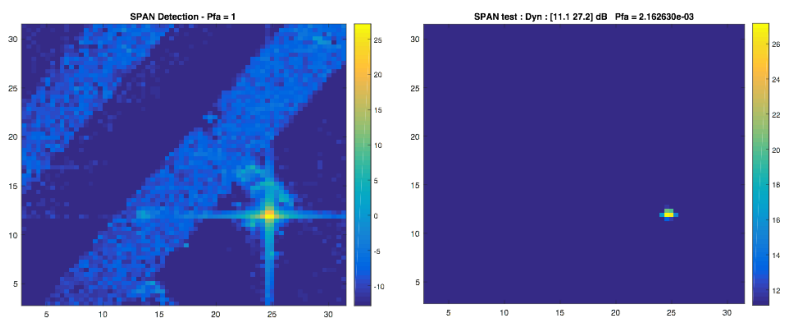

Fig. 10. Left: SPAN detection dest, $P_{f a}=1$. Right: Thresholded SPAN detection test, $P_{f a}=2.610^{-3}$.
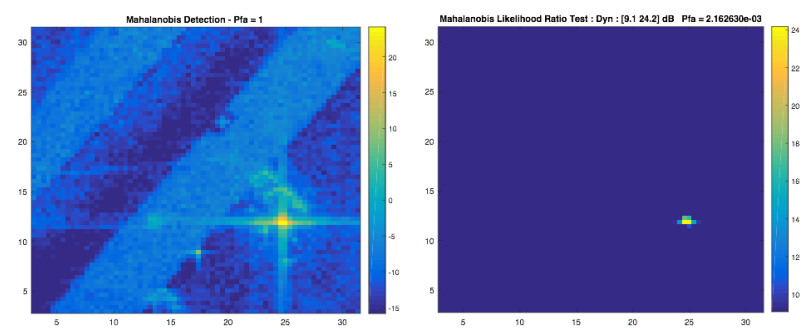

Fig. 11. Left: Mahalanobis-TE detection dest, $P_{f a}=1$. Right: Thresholded Mahalanobis detection test, $P_{f a}=2.610^{-3}$. 


\section{REFERENCES}

[1] R. S. G. W. G. Carrara and R. M. Majewski, Spotlight Synthetic Aperture Radar: Signal Processing Algorithms, U. S. Norwood, Ed. Artech House Publishers, 1995.

[2] High Resolution Radar Imaging. Artech House Publishers, 1983.

[3] M. Soumekh, Synthetic Aperture Radar Signal Processing with MATLAB Algorithms. New York: John Wiley and Sons, 1999.

[4] D. Tao, S. N. Anfinsen, and C. Brekke, "Robust cfar detector based on truncated statistics in multiple-target situations," Geoscience and Remote Sensing, IEEE Transactions on, vol. 54, no. 1, pp. 117-134, Jan 2016.

[5] R. Durand, G. Ginolhac, L. Thirion, and P. Forster, "New sar processor based on matched subspace detectors," Aerospace and Electronic Systems, IEEE Transactions on, vol. 45, no. 1, pp. 221-236, Jan 2009.

[6] E. P. Simoncelli, W. T. Freeman, E. H. Adelson, and D. J. Heeger, "Shiftable multiscale transforms," Information Theory, IEEE Transactions on, vol. 38, no. 2, pp. 587-607, March 1992.

[7] M. Unser, N. Chenouard, and D. V. D. Ville, "Steerable pyramids and tight wavelet frames in," Image Processing, IEEE Transactions on, vol. 20, no. 10, pp. 2705-2721, Oct 2011.

[8] E. Candès, L. Demanet, D. Donoho, and L. Ying, "Fast discrete curvelet transforms," Multiscale Modeling \& Simulation, vol. 5, no. 3, pp. 861-899, 2006. [Online]. Available: http://dx.doi.org/10.1137/05064182X

[9] J. Bertrand and P. Bertrand, "The concept of hyperimage in wide-band radar imaging," Geoscience and Remote Sensing, IEEE Transactions on, vol. 34, no. 5, pp. 1144-1150, Sep. 1996.

[10] J. P. Ovarlez, L. Vignaud, J. C. Castelli, M. Tria, and M. Benidir, "Analysis of sar images by multidimensional wavelet transform," IEE Proceedings - Radar, Sonar and Navigation, vol. 150, no. 4, pp. 234-241, Aug. 2003.

[11] M. Tria, J. P. Ovarlez, L. Vignaud, J. C. Castelli, and M. Benidir, "Discriminating real objects in radar imaging by exploiting the squared modulus of the continuous wavelet transform," IET Radar, Sonar and Navigation, vol. 1, no. 1, pp. 27-37, Feb. 2007.

[12] M. Duquenoy, J. P. Ovarlez, L. Ferro-Famil, E. Pottier, and L. Vignaud, "Scatterers characterisation in radar imaging using joint time-frequency analysis and polarimetric coherent decompositions," IET Radar, Sonar and Navigation, vol. 4, no. 3, pp. 384-402, June 2010.

[13] J. Bertrand, P. Bertrand, and J. P. Ovarlez, "Dimensionalized wavelet transform with application to radar imaging," in Proc. IEEE International Conference on Acoustics, Speech, and Signal Processing (ICASSP'91), vol. 4, Toronto, Canada, Apr. 1417, 1991, pp. 2909-2912.

[14] L. Cohen, "Time-frequency distributions-a review," Proceedings of the IEEE, vol. 77, no. 7, pp. 941-981, Jul 1989.

[15] M. Tello, C. Lopez-Martinez, and J. J. Mallorqui, "A novel algorithm for ship detection in sar imagery based on the wavelet transform," IEEE Geoscience and Remote Sensing Letters, vol. 2, no. 2, pp. 201-205, April 2005.
[16] I. Reed and X. Yu, "Adaptive multiple-band cfar detection of an optical pattern with unknown spectral distribution," Acoustics, Speech and Signal Processing, IEEE Transactions on, vol. 38, no. 10, pp. 1760-1770, Oct 1990.

[17] J. Frontera-Pons, M. A. Veganzones, F. Pascal, and J. P. Ovarlez, "Hyperspectral anomaly detectors using robust estimators," Selected Topics in Applied Earth Observations and Remote Sensing, IEEE Journal of, vol. 9, no. 2, pp. 720-731, Feb 2016.

[18] E. Ollila, D. E. Tyler, V. Koivunen, and H. V. Poor, "Complex elliptically symmetric distributions: Survey, new results and applications," Signal Processing, IEEE Transactions on, vol. 60, no. 11, pp. $5597-5625$, nov. 2012.

[19] F. Pascal, Y. Chitour, J.-P. Ovarlez, P. Forster, and L. P., "Covariance structure maximum likelihood estimates in compound gaussian noise: Existence and algorithm analysis," Signal Processing, IEEE Transactions on, vol. 56, no. 1, pp. 34-38, January 2008.

[20] Y. Chen, A. Wiesel, and A. O. Hero, "Robust shrinkage estimation of high-dimensional covariance matrices," Signal Processing, IEEE Transactions on, vol. 59, no. 9, pp. 4097-4107, 2011.

[21] F. Pascal, Y. Chitour, and Y. Quek, "Generalized robust shrinkage estimator and its application to stap detection problem," Signal Processing, IEEE Transactions on, vol. 62, no. 21, pp. 5640-5661, Nov 2014.

[22] E. Ollila and D. E. Tyler, "Regularized m-estimators of scatter matrix," Signal Processing, IEEE Transactions on, vol. 62, no. 22, pp. 6059-6070, Nov 2014.

[23] F. C. Robey, D. R. Fuhrmann, E. J. Kelly, and R. Nitzberg, "A CFAR adaptive matched filter detector," Aerospace and Electronic Systems, IEEE Transactions on, vol. 28, no. 1, pp. 208216, Jan 1992.

[24] S. Kraut, L. Scharf, and L. M. Whorter, "Adaptive Subspace Detector," Signal Processing, IEEE Transactions on, vol. 49, no. 1, pp. 1-16, Jan 2001.

[25] M. S. Greco and A. D. Maio, Eds., Modern Radar Detection Theory. SciTech Publishing, Jan 2016. 